\title{
Burden of Sensitive Skin (BoSS) Questionnaire and Current Perception Threshold: Use as Diagnostic Tools for Sensitive Skin Syndrome
}

\author{
Helena POLENA ${ }^{1}$, Marlène CHAVAGNAC-BONNEVILLE ${ }^{1}$, Laurent MISERY2,3 and Michèle SAYAG ${ }^{1}$ \\ ${ }^{1}$ Research and Development Department, NAOS, Lyon, '2Laboratoire Interactions Epitheliums Neurones (LIEN), University of Brest, and \\ ${ }^{3}$ Department of Dermatology, University Hospital of Brest, Brest, France
}

The assessment of sensitive skin syndrome, characterized by subjective unpleasant sensations, remains a challenge, since there is no international consensus on the best diagnostic tools. This study evaluated the combination of the Burden of Sensitive Skin (BoSS) questionnaire and the current perception threshold as diagnostic tools for sensitive skin syndrome, and the relationship between BoSS and the subjects' smoking status, phototype and skin type. A total of 100 women completed the Boss questionnaire, and current perception threshold was measured on the face. The Boss score was significantly higher in the self-reported sensitive skin group compared with the non-sensitive skin group ( 25.61 vs $14.05 ; p<0.001$ ), and in non-smokers vs smokers $(23.00$ vs $18.37 ; p<0.05)$. In addition, the current perception threshold values were similar between the sensitive and non-sensitive groups. These results suggest that BoSS is a better diagnostic tool for sensitive skin syndrome than the current perception threshold, and that smokers less frequently have sensitive skin than do non-smokers.

Key words: burden; sensitive skin; sensory perception; neurometer; diagnostic; smoking; Boss.

Accepted Oct 14, 2021; Epub ahead of print Oct 14, 2021

Acta Derm Venereol 2021; 101: adv00606.

Corr: Helena Polena, NAOS, Research and Development Department, 75 cours Albert Thomas, FR-69003 Lyon, France. E-mail: helena.polena@ naos.com

$\mathrm{S}$ ensitive skin syndrome (SSS) is a common skin condition, defined by the occurrence of unpleasant sensory perceptions, such as stinging, burning, tingling, or itching in response to physical, thermal, chemical, hormonal, psychological, or other stimuli that normally do not provoke such sensations (1). Sensitive skin may have a normal appearance or be accompanied by erythema. All body parts can be affected, but the face is the most frequently involved. The prevalence of SSS is approximately 39\% among European populations, with more women than men claiming to have sensitive skin (2).

While SSS places a considerable burden on patients (3), its physiopathology is not well understood. Two main hypotheses for the physiopathology of SSS have been proposed: an alteration in the epidermal barrier function and a neurosensory dysfunction (4). Recently, SSS was described as a small-fibre neuropathy characterized by damage to the small cutaneous $\mathrm{A} \delta$ and $\mathrm{C}$ nerve fibres

\section{SIGNIFICANCE}

The diagnosis of sensitive skin is difficult for dermatologists, since it is characterized by subjective symptoms, and because no international consensus exists on the best diagnostic tools. This study, performed on 100 women, demonstrates that the Burden of Sensitive Skin (BoSS) questionnaire, evaluating the burden of subjects with sensitive skin, can also be used by dermatologists as a diagnostic tool, and that smokers present less sensitive skin on the face than do non-smokers. However, the problem of objective assessment of sensitive skin remains unsolved.

(5), especially unmyelinated C-fibres (6). Furthermore, a study demonstrated that, compared with control subjects, patients with SSS had significantly higher scores for neuropathic pain and showed a significantly lower heat pain threshold, measured with a quantitative sensory test, suggesting that their unmyelinated $\mathrm{C}$ fibres were damaged (7).

Internationally, no consensus exists on how to assess and diagnose SSS, as the assessment of subjective phenomenon is always difficult. Because SSS presents with mainly subjective symptoms, an assessment using patient-reported scales might be the most appropriate $(6,8)$. Consequently, several questionnaires have been developed to assess sensitive skin and how it affects patients' quality of life. However, only a few of the questionnaires have been validated. Among these are the Sensitive Scale, with its 10-item version (SS-10, correlating with the Dermatology Quality of Life Index) (9), the $3 \mathrm{~S}$ questionnaire for sensitive scalp (10), and the Burden of Sensitive Skin (BoSS), developed in 2018 following a rigorously validated methodology (11).

Nevertheless, while subjective methods are specific, they show low reproducibility, as they rely on the patient's perception. The combined use of 2 different SSS diagnostic methods may be a more robust alternative for diagnosis. The stinging test, with either lactic acid or capsaicin, is frequently used to assess SSS, but is specific for the substance applied and the timing of application, hence the test is not representative of the global skin sensitivity of the patient's skin (8).

Sensitivity evaluations using the current perception threshold (CPT) enable further insight into the underlying mechanisms by distinguishing the type of nerve fibres involved. The Neurometer ${ }^{\circledR} \mathrm{CPT}^{\circledR}$ device (Neurotron Inc., 
Aurora, CO, USA) is an electrical current generator that measures the sensory threshold non-invasively on the skin. This painless procedure assesses the functional integrity of sensory fibres by applying transcutaneous electrical stimuli through 2 electrodes and measuring the CPT. The electrical currents concomitantly excite large and small sensory nerve fibres and allow their differentiation from each other. The frequencies produced by the Neurometer ${ }^{\mathbb{B}}$ $\mathrm{CPT}^{\circledR}$ provide selective stimulation of 3 subsets of nerve fibres: the 2,000-Hz current stimulates large myelinated A $\beta$ fibres (touch and pressure sensation), the $250-\mathrm{Hz}$ current stimulates small myelinated A $\delta$ fibres (temperature, pressure, fast pain, and prickling itch sensation), and the $5-\mathrm{Hz}$ current stimulates unmyelinated C-fibres (temperature, slow pain, and burning itch sensation).

The aim of this study was to determine whether the BoSS questionnaire, the CPT, or a combination of both are valid diagnostic tools for SSS. Moreover, this study aimed to investigate a potential correlation of the test results with the self-reported severity of facial skin sensitivity and patients' smoking status, phototype, and skin type.

\section{MATERIALS AND METHODS}

\section{Study subjects and design}

This explorative open study was conducted in accordance with the French and European Guidelines for Good Clinical Practice, the recommendations of the International Council for Harmonization of Technical Requirements for Pharmaceuticals for Human Use, the Declaration of Helsinki 1964 and its later amendments, and the laws and regulations currently in force in France. According to the local and European regulatory guidelines, this type of study does not require approval from local ethics committees. Written informed consent was obtained from all subjects.

In order to include an homogeneous population of women with sensitive skin, according to Misery et al. (12), the inclusion criteria were: Caucasian type, female sex, age between 25 and 50 years, smoker ( $50 \%$ of the cohort), and presenting with phototype I to III according to the Fitzpatrick scale. The standard exclusion criteria were as follows: pregnant or breastfeeding, patients undergoing medical treatment, medical history of allergy to cosmetics or medicines, exposure to sunlight within 4 weeks preceding the study, skin irritation on the face, and application of products to the face on the day of the test (except for usual cleansers).

Among the 104 women reporting having SSS, screened from the CPP Initiative/Dermatec database (Tassin La Demi-Lune, France), who qualified for the current study, according to their self-reported sensitive skin, smoking behaviour, phototype and skin type, 102 participants were enrolled for the study between March 2019 and April 2019. A total of 100 subjects completed the study and were included in the analysis.

During a single visit to the CPP Initiative/Dermatec investigation centre, the subjects completed the BoSS questionnaire, and sensory perception measurements were performed, as described below.

\section{BoSS questionnaire}

The BoSS questionnaire, previously described in detail by Misery et al. (11), was completed by all subjects. It comprises 14 questions grouped in 3 dimensions:
- Self-care (finding wool uncomfortable, impact on clothing purchase, on choice of laundry detergent, on choice of soap, on choice of clothes in the morning, on choice of cosmetics, and on choice of jewellery).

- Daily life (restricted food, impact on choice of leisure activities, linked to air conditioning, and pollution).

- Appearance (blushing with emotion, blushing after physical activity, and being annoyed when photographed).

The BoSS questionnaire allows the subject to self-assess the burden of their sensitive skin. The total score is calculated by summing the scores for each of the 14 questions using the following scale: $0=$ never, $1=$ rarely, $2=$ sometimes, $3=$ often, $4=$ constantly. The total score ranges from 0 to 56 .

\section{Measurement of sensory perception}

Sensory perception was determined as the CPT measured with the Neurometer ${ }^{\circledR} \mathrm{CPT}^{\mathbb{\circledR}} / \mathrm{C}$ (Neurotron Inc., Denver, CO, USA) under environmentally controlled conditions $\left(20 \pm 2^{\circ} \mathrm{C}, 50 \pm 10 \%\right.$ relative humidity), as previously described (13). Measurements were performed on the cheek within the innervation area of the maxillary trigeminal nerve on both sides of the face.

\section{Statistical and descriptive analysis}

Continuous variables were normally distributed as the mean \pm standard deviation. The BoSS score data were presented as box plots with whiskers represented either the maximum values or 1.5 times the interquartile range of the data, whichever was smaller.

Comparisons of the BoSS scores for the 3 dimensions and the total score between different levels of binary qualitative variables (i.e. skin sensitivity, smoking) were performed using the Student's test or Wilcoxon test, similar to the comparisons of the CPT measurements (at 2,000, 250 and $5 \mathrm{~Hz}$ ). The BoSS scores (the score from the 3 dimensions and total score) of qualitative variables having more than 2 levels (i.e. phototype and facial skin type) were tested using 1-way analysis of variance followed by pairwise post-hoc Tukey tests. A potential association between the BoSS scores and CPT values was assessed using Pearson's correlation coefficient. The level of statistical significance was defined as a $p$-value $<0.05$.

An exploratory analysis was performed using principal component analysis (PCA) of the data obtained on both sides of the subjects' faces $(n=200)$ characterized by 6 standardized variables (the scores of each dimension of the BoSS questionnaire and the CPT values at 2,000, 250 and $5 \mathrm{~Hz}$ ) in order to reduce the dimensionality of the graph from 6 to 2 axes while maintaining the highest level of variance. The main 2 axis of the PCA described $71 \%$ of the total inertia (39\% and $32 \%$, respectively) and were retained in order to compute a graphical visualization of the space occupied by the subjects defined by these 2 axes. Subjects were grouped according to their self-reported facial skin sensitivity and their smoking status.

PCA and statistical (Student, Shapiro and Wilcoxon) tests were performed with R software (version 5.3.2) (https://www.R-project. org/) using the package ade4.

\section{RESULTS}

\section{Subjects' characteristics}

A total of 100 female subjects aged 24 to 50 years (mean age $36.7 \pm 7.6$ years) completed the study. Sixty percent presented with dry to mixed dry facial skin and $40 \%$ with 

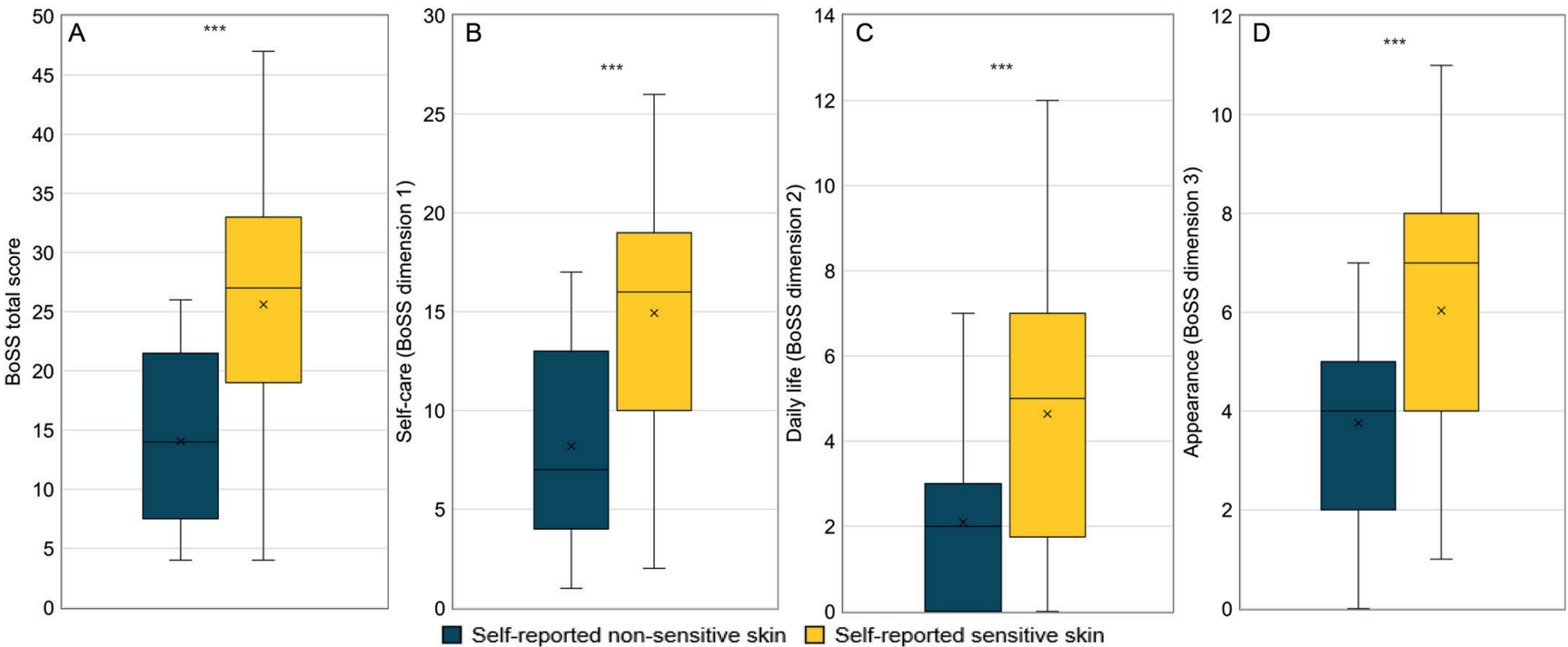

Fig. 1. Burden of Sensitive Skin (BoSS) scores according to the self-reported facial skin sensitivity of the subjects ( $n=100$ ). (A) BoSS total score; (B) self-care score; (C) daily life score; (D) appearance score $(* * * p<0.001)$. Data are presented as box plots representing the lower quartile, the median (black line) and the upper quartile. Crosses represent mean values.

normal to combination skin, and $64 \%$ were phototype III, while the remaining $46 \%$ were phototype I-II. Among all subjects, $59 \%$ self-reported sensitive facial skin $(n=59)$, and $54 \%$ were smokers $(n=49)$.

\section{Burden of Sensitive Skin questionnaire}

The BoSS mean total score for the subjects with selfreported sensitive facial skin was 25.61, and the score for the subjects without sensitive skin was $14.05(p<0.001)$ (Fig. 1). Subjects with self-reported sensitive facial skin also had higher scores for each of the 3 BoSS dimensions compared with self-reported non-sensitive skin subjects: self-care (14.93 vs 8.20 ), daily life (4.64 vs 2.10$)$, and appearance (6.03 vs 3.76), all $p<0.001$ (Fig. 1).
A statistically significant difference was also observed between the BoSS total score of 23.00 in non-smokers and that of 18.37 in smokers ( $p<0.05$ ), which was mostly related to the significantly higher score in the appearance dimension of 5.72 vs 4.37 , respectively ( $p<0.01$; Fig. 2). The results did not show a difference in the BoSS score between different phototypes or skin types, regardless of the dimension.

\section{Measurements of sensory perception}

The distribution of the CPT values for the 3 frequencies employed was homogeneous between the self-reported sensitive skin group and self-reported non-sensitive skin group (Table I). However, the self-reported non-sensitive
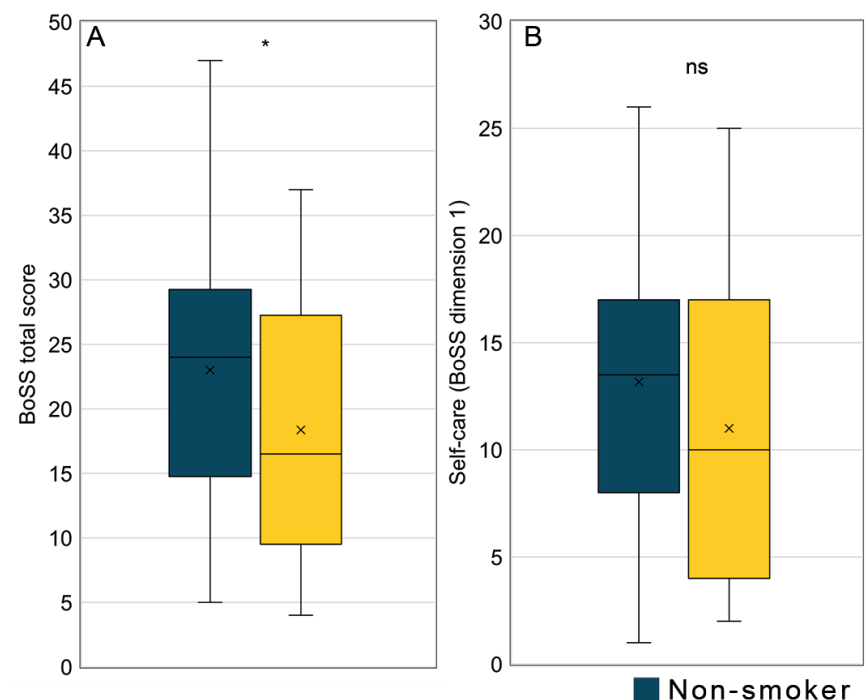
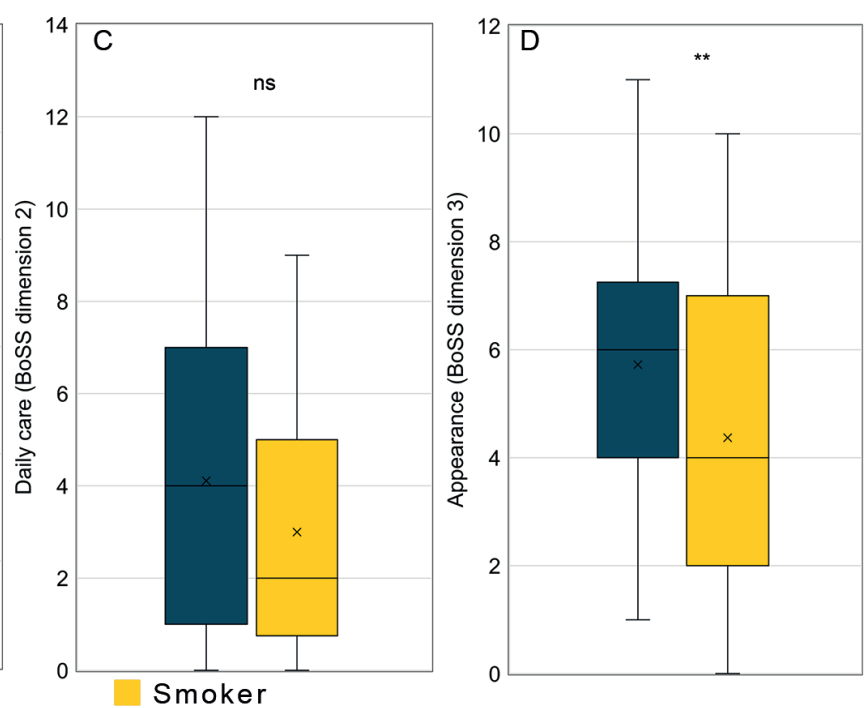

Fig. 2. Burden of Sensitive Skin (Boss) scores according to the smoking status of subjects ( $\boldsymbol{n}=100)$. (A) BoSS total score; (B) self-care score; (C) daily life score; (D) appearance score (ns: $p>0.05 ; * p<0.05 ; * * p<0.01$ ). Data are presented as box plots representing the lower quartile, the median (black line) and the upper quartile. Crosses represent mean values. 
Table I. Analysis of the current perception thresholds at 2,000, 250 and $5 \mathrm{~Hz}$ and the Burden of Sensitive Skin (BoSS) score in subjects with self-reported sensitive and non-sensitive skin for both sides of their faces $(n=200)$

\begin{tabular}{|c|c|c|c|c|c|c|c|c|c|c|}
\hline & \multicolumn{5}{|c|}{ Self-reported sensitive skin $(n=118)$} & \multicolumn{5}{|c|}{ Self-reported non-sensitive skin $(n=82)$} \\
\hline & \multicolumn{3}{|c|}{$\mathrm{CPT}$ values, $\mathrm{Hz}$} & \multirow{2}{*}{$\begin{array}{l}\triangle \mathrm{CPT}, \mathrm{Hz} \\
(250-5)\end{array}$} & \multirow{2}{*}{$\begin{array}{l}\text { BoSS } \\
\text { Total score }\end{array}$} & \multicolumn{3}{|c|}{$\mathrm{CPT}$ values, $\mathrm{Hz}$} & \multirow{2}{*}{$\begin{array}{l}\triangle \mathrm{CPT}, \mathrm{Hz} \\
(250-5)\end{array}$} & \multirow{2}{*}{$\begin{array}{l}\text { BoSS } \\
\text { Total score }\end{array}$} \\
\hline & 2,000 & 250 & 5 & & & $2,000 \mathrm{~Hz}$ & 250 & 5 & & \\
\hline Mean (SD) & $81.9(38.2)$ & $12.1(8.9)$ & $5.3(4.6)$ & $6.8(6.6)$ & $25.6(9.5)$ & $78.3(36.2)$ & $11.7(9.5)$ & $4.9(4.1)$ & $6.8(8.9)$ & $14(7.1)$ \\
\hline Median & 85.0 & 9.5 & 3.0 & 6.5 & 27 & 75.0 & 9.5 & 3.0 & 3.5 & 14 \\
\hline Min-Max & $12-226$ & $1.7-40$ & $2-27$ & & $4-47$ & $21-171$ & $2-40$ & $2-23$ & & $4-26$ \\
\hline \multicolumn{11}{|c|}{ CTP values repartition, $n(\%)$} \\
\hline Inf min* & $18(15)$ & $15(13)$ & $0(0)$ & $46(39)$ & & $14(17)$ & $12(15)$ & $0(0)$ & $40(49)$ & \\
\hline Range $\min * *$ & $84(71)$ & $78(66)$ & $105(89)$ & & & $57(70)$ & $50(61)$ & $74(90)$ & & \\
\hline Range $\max ^{* * *}$ & $16(14)$ & $25(21)$ & $13(11)$ & & & $11(13)$ & $20(24)$ & $8(10)$ & & \\
\hline Sup max**** & $0(0)$ & $0(0)$ & $0(0)$ & & & $0(0)$ & $0(0)$ & $0(0)$ & & \\
\hline$*$ & $<40$ & $<4$ & $<1$ & $\Delta \mathrm{CPT}<3$ & & & & & & \\
\hline$* *$ & $40-117$ & $4-17$ & $1-9$ & & & & & & & \\
\hline$* * *$ & $118-244$ & $18-52$ & $10-38$ & & & & & & & \\
\hline$* * * *$ & $>244$ & $>52$ & $>38$ & & & & & & & \\
\hline
\end{tabular}

CPT: current perception threshold; SD: standard deviation; Inf: inferior; min: minimum; max: maximum; Sup: superior.

skin cohort presented similar CPT values between the frequencies of $250 \mathrm{~Hz}$ and $5 \mathrm{~Hz}$ (49\% of the subjects). To observe this difference, the delta CPT was computed for both groups. Even if the means were similar, the median delta CPT was lower in the self-reported non-sensitive skin group due to similar values at $250 \mathrm{~Hz}$ and $5 \mathrm{~Hz}$ for half of the group. The results did not show a difference in the CPT measurements and the smoking status of the subjects, regardless of the frequency employed.
Burden of Sensitive Skin questionnaire and measurements of sensory perception

No significant association was found between the BoSS scores and CPT measurements, regardless of the frequency employed.

The 2 main axes of the PCA accumulated $71 \%$ of the total inertia. The first PCA axis (39\% of total inertia) was represented mainly by the variables related to the BoSS questionnaire. This values on this axis increased with
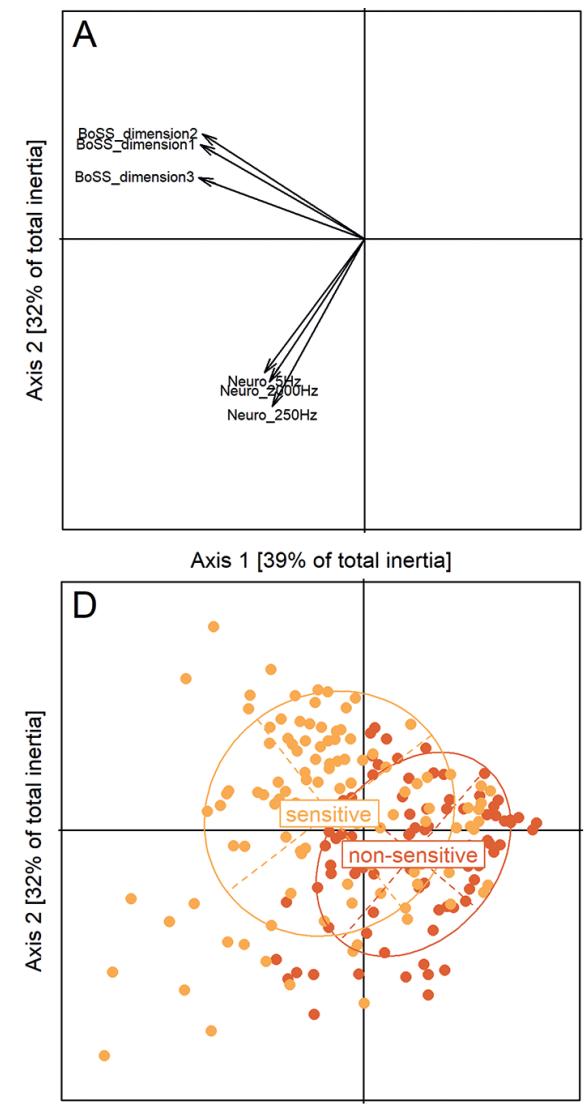

Axis $1[39 \%$ of total inertia]

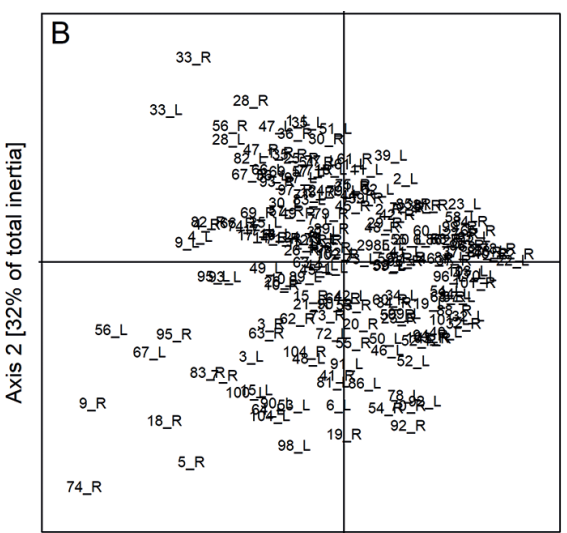

Axis 1 [39\% of total inertia]

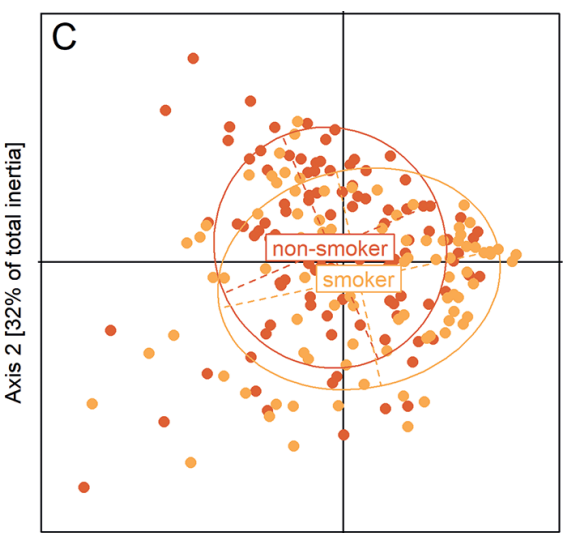

Axis $1[39 \%$ of total inertia]

Fig. 3. Principal component analysis (PCA) with axis 1 vs axis 2 characterized by the 3 dimensions of Burden of Sensitive Skin (BoSS) scores and the 3 current perception threshold (CPT) frequencies (Neuro_2000Hz, Neuro_250Hz, Neuro_5Hz (Neurometer ${ }^{\circledR}$ CPT ${ }^{\circledR} / \mathrm{C}$ (Neurotron Inc., Denver, CO, USA)) according to smoking status and facial skin sensitivity. (A) Solid arrows indicate the direction and weighing of vectors representing the 6 considered variables. (B) The subjects projected on the PCA plane (dots) are represented by their number and the face side (right or left). (C) The subjects are grouped according to their smoking status, and (D) to the presence of facial sensitive skin. 
decreasing BoSS scores for the 3 variables (Fig. 3A). The second axis (32\% of total inertia) was represented mainly by the 3 variables related to the CPT measurements. The values on this axis increased with decreasing CPT values at the 3 frequencies (Fig. 3A). The distribution of subjects in this 2-dimensional space showed that the BoSS scores were lower in smokers than in non-smokers (Fig. 3C). Indeed, the coordinates along the first PCA axis were higher for smokers than for non-smokers. The results also showed that smokers had higher CPT values, with coordinates along the second PCA axis lower than those of non-smokers. Similarly, self-reported facial skin sensitivity was associated with higher BoSS scores than those in subjects without sensitive skin (Fig. 3D).

\section{DISCUSSION}

To the best of our knowledge, this is the first study using CPT measurements in combination with the BoSS as a diagnostic tool for SSS. Three conclusions can be drawn from these results. First, this study showed an association between the BoSS scores and the self-report of facial skin sensitivity, which might enable us to distinguish between sensitive and non-sensitive skin. Secondly, the results suggest that either smokers less frequently have SSS than non-smokers. Thirdly, no significant association was found between CPT values and self-reported sensitive skin, BoSS scores, or smoking status, regardeless of the CPT frequency employed. Interestingly, a trend toward similar CPT values at frequencies of 250 and $5 \mathrm{~Hz}$ was found in subjects without facial sensitive skin, and higher CPT values were observed in smokers by PCA analysis, compared with non-smokers.

The BoSS questionnaire measures the burden of SSS, which is associated with the severity of the disease, but the current study shows that it may also serve to diagnose SSS. In our initial study, the mean BoSS score in the very sensitive facial skin group corresponded to a BoSS score of $\geq 23$ (11). In the current study, the mean BoSS score of the self-reported sensitive skin group was 25.6, suggesting that these subjects can be considered as having a very sensitive skin.

Moreover, the findings of the current study indicate an association between absence of smoking and SSS. The smoking status of subjects with SSS was also investigated by Falcone et al. (14), who demonstrated that 258 women who smoked at the time of the study or had a history of smoking had an increased, albeit not significantly increased, risk of sensitive skin compared with non-smoking women. A recent study performed in China on 1,598 subjects reported more smokers with sensitive skin (15). In the current study, the subjects were only asked whether they currently smoked and not whether they had smoked in the past, which might explain the difference in the results. An explanation of the reduction in SSS by smoking could be the thickness of the stratum corneum in smokers, although data in the literature are controversial. In one study, the thickness of the stratum corneum correlated negatively with the number of years smoking, whereas no correlation with smoking status was found in 71 men and women (16). Another study demonstrated that 98 men who had smoked for at least 15 years had a thicker stratum corneum on their cheeks, but not on their temple, abdomen, dorsal forearm, or non-sun-exposed upper inner arm, compared with men who had never been smokers (17). Another hypothesis could be an adaptation of the skin to chronic exposure to smoke, inducing a reduction in skin sensitivity to avoid overreaction. Finally, nicotine, the major compound of cigarettes, is known to have anti-inflammatory properties through inhibition of prostaglandin synthesis $(18,19)$.

No variations in the BoSS scores were observed in relation to phototypes or skin types. Regarding the skin phototype, some studies have associated sensitive skin with fair skin $(12,14,20)$, possibly due to a thinner epidermis than in more pigmented skin (16). However, the association between sensitivity and fair skin remains controversial, as other studies could not confirm an association of sensitive skin with skin phototype or ethnicity variations (20-23). While the current results do not show an association, subjects with phototypes IV to VI were excluded. The results showed no association between self-reported sensitive skin and dry, normal, or combination skins, in agreement with the systemic review by Richters et al. (24), whereas dry skin has been described as more sensitive than normal, combination, or oily skins $(12,20)$.

No association of CPT values with self-reported sensitive skin was observed in the current study. Since damage to small cutaneous $\mathrm{A} \delta$ and $\mathrm{C}$ nerve fibres, especially unmyelinated C-fibres (6), has been described in sensitive skin (5), we expected a significant decrease in the CPT at $5 \mathrm{~Hz}$ in sensitive skin compared with non-sensitive skin. This has been described in several studies $(13,25$, $27,28)$. In some reports, CPT was also reduced at 250 $\mathrm{Hz}(13,27,28)$. In previous studies using CPT assessment, the stinging test (mostly with lactic acid) has been used to discriminate sensitive skin. Herein, self-reported sensitive skin and the BoSS questionnaire were used to identify sensitive skin more adequately $(13,25,27,28)$. Some possible limitations of the current study are the number and age of the subjects, as well as the skin types and phototypes included in the cohort.

The diagnosis of SSS is difficult, as it is commonly based on subjective symptoms, without defined individual thresholds. Nevertheless, since the BoSS questionnaire was able to identify SSS in this study, a score calculated from the BoSS that indicates the presence of sensitive skin could be useful for future studies. As SSS is a subjective phenomenon, the performance of cumbersome objective tests may be of limited value. 
In conclusion, this study suggests that the smoking status of the patients should be taken into consideration during diagnosis of SSS. In addition, the BoSS questionnaire, more than a SSS burden evaluation questionnaire, can also be considered as a good diagnostic tool for SSS, whereas CPT measurements could be more useful in identifying the absence of SSS, as performed in subjects with diabetes (29). Additional investigations are needed in larger and more diverse populations to propose a cut-off score. Finally, further studies on the correlation between the BoSS score and objective measurements of skin sensitivity should be performed to improve our understanding of SSS.

\section{ACKNOWLEDGEMENTS}

The authors thank CPP Initiatives/Dermatec (Tassin la DemiLune, France) for the management of the clinical study, Bruno Vandelanotte (Neurotron Inc., Brugge, Belgium) and Félix Giraud (NAOS, Aix-en-Provence, France) for providing technical advice. This study was funded by NAOS (Bioderma - Institut Esthederm - Etat Pur).

Conflicts of interest. HP, MCB and MS are employees of NAOS. LM reported personal fees from NAOS during the conduct of the study.

\section{REFERENCES}

1. Misery L, Ständer S, Szepietowski JC, Reich A, Wallengren J, Evers AWM, et al. Definition of sensitive skin: an expert position paper from the special interest group on sensitive skin of the international forum for the study of itch. Acta Derm Venereol 2017; 97: 4-6.

2. Misery L, Boussetta S, Nocera T, Perez-Cullell N, Taieb C. Sensitive skin in Europe. J Eur Acad Dermatology Venereol 2009; 23: 376-381.

3. Farage MA, Maibach HI. Sensitive skin: closing in on a physiological cause. Contact Dermatitis 2010; 62: 137-149.

4. Talagas M, Misery L. Role of keratinocytes in sensitive skin. Front Med 2019; 6: 1-7.

5. Buhé V, Vié K, Guéré C, Natalizio A, Lhéritier C, Le GallIanotto C, et al. Pathophysiological study of sensitive skin. Acta Derm Venereol 2016; 96: 314-318.

6. Huet F, Misery L. Sensitive skin is a neuropathic disorder. Exp Dermatol 2019; 28: 1470-1473.

7. Huet F, Dion A, Batardière A, Nedelec AS, Le Caër F, Bourgeois $P$, et al. Sensitive skin can be small fibre neuropathy: results from a case-control quantitative sensory testing study. $\mathrm{Br}$ J Dermatol 2018; 179: 1157-1162.

8. Misery L, Loser K, Ständer S. Sensitive skin. J Eur Acad Dermatology Venereol 2016; 30: 2-8.

9. Misery L, Jean-Decoster C, Mery S, Georgescu V, Sibaud V. A new ten-item questionnaire for assessing sensitive skin: the sensitive scale-10. Acta Derm Venereol 2014; 94: 635-639.

10. Misery L, Rahhali N, Ambonati M, Black D, Saint-Martory C, Schmitt AM, et al. Evaluation of sensitive scalp severity and symptomatology by using a new score. J Eur Acad Derma- tology Venereol 2011; 25: 1295-1298.

11. Misery L, Jourdan E, Abadie S, Ezzedine K, Brenaut E, Huet $F$, et al. Development and validation of a new tool to assess the Burden of Sensitive Skin (BoSS). J Eur Acad Dermatology Venereol 2018; 32: 2217-2223.

12. Misery L, Jourdan E, Huet F, Brenaut E, Cadars B, Virassamynaïk $S$, et al. Sensitive skin in France: a study on prevalence, relationship with age and skin type and impact on quality of life. J Eur Acad Dermatology Venereol 2018; 32: 791-795.

13. Kim SJ, Lim SU, Won YH, An SS, Lee EY, Moon SJ, et al. The perception threshold measurement can be a useful tool for evaluation of sensitive skin. Int J Cosmet Sci 2008; 30: 333-337.

14. Falcone D, Richters RJH, Uzunbajakava NE, van Erp PEJ, van de Kerkhof PCM. Risk factors associated with sensitive skin and potential role of lifestyle habits: a cross-sectional study. Clin Exp Dermatol 2017; 42: 656-658.

15. Chan MKT, Sayag M, Chavagnac-Bonneville M, Taieb C, Misery L. Sensitive skin in China: characteristics and burden. J Eur Acad Dermatol Venereol 2021; 35: e436-e439.

16. Sandby-Møller J, Poulsen T, Wulf HC. Epidermal thickness at different body sites: relationship to age, gender, pigmentation, blood content, skin type and smoking habits. Acta Derm Venereol 2003; 83: 410-413.

17. Knuutinen A, Kallioinen M, Vähäkangas K, Oikarinen A. Smoking and skin: a study of the physical qualities and histology of skin in smokers and non-smokers. Acta Derm Venereol 2002; 82: 36-40.

18. Baron JA. Beneficial effects of nicotine and cigarette smoking: the real, the possible and the spurious. British Medical Bulletin 1996; 52: 58-73.

19. Misery L. Nicotine effects on skin: Are they positive or negative. Exp Dermatol 2004; 13: 665-760.

20. Misery L, Sibaud V, Merial-Kieny C, Taieb C. Sensitive skin in the American population: prevalence, clinical data, and role of the dermatologist. Int J Dermatol 2011; 50: 961-967.

21. Jourdain R, De Lacharrière O, Bastien P, Maibach HI. Ethnic variations in self-perceived sensitive skin: epidemiological survey. Contact Dermatitis 2002; 46: 162-169.

22. Farage MA. How do perceptions of sensitive skin differ at different anatomical sites? An epidemiological study. Clin Exp Dermatol 2009; 34: e521-523.

23. Misery L, Myon E, Martin N, Consoli S, Boussetta S, Nocera T, et al. Sensitive skin: psychological effects and seasonal changes. J Eur Acad Dermatology Venereol 2007; 21: 620-628.

24. Richters R, Falcone D, Uzunbajakava N, Verkruysse W, Van Erp $P$, Van De Kerkhof P. What is sensitive skin? A systematic literature review of objective measurements. Skin Pharmacology and Physiology 2015; 28: 75-83.

25. Ham H, An SM, Lee EJ, Lee E, Kim HO, Koh JS. Itching sensation and neuronal sensitivity of the skin. Ski Res Technol 2016; 22: 104-107.

26. Sun L, Wang $X$, Zhang Y, Wang T, Li X, Ma Y. The evaluation of neural and vascular hyper-reactivity for sensitive skin. Ski Res Technol 2016; 22: 381-387.

27. Li S, Wang X, Gao Y, Chen S, Liu X, Wang H, et al. CPT, the main test method of skin neuronal sensitivity. Cutan Ocul Toxicol 2015; 34: 208-211.

28. Lee E, An S, Lee TR, Kim HK. Development of a novel method for quantitative evaluation of sensory skin irritation inhibitors. Ski Res Technol 2009; 15: 464-469.

29. Putz S, Gtabák A, Toth N, Istenes I, Nemeth N, Gandhi RA, et al. Noninvasive evaluation of neural impairment in subjects with impaired glucose tolerance. Diabetes Care 2009; 32: 181-183, 